\title{
Relationship Between the Extent of Endolymphatic Hydrops and the Severity and Fluctuation of Audiovestibular Symptoms in Patients With Menière's Disease and MRI Evidence of Hydrops
}

\author{
Claudia Jerin, Sonja Floerke, Rebecca Maxwell, and Robert Gürkov \\ German Center for Vertigo and Balance Disorders and Department of Otorhinolaryngology_Head and Neck Surgery, \\ Grosshadern Medical Center, University of Munich, Munich, Germany
}

\begin{abstract}
Objective: To characterize the short-, middle-, and longterm occurrence of vertigo attacks in a large population of Menière's disease (MD) and to investigate the relationship between the extent of endolymphatic hydrops (ELH) and the severity of audiovestibular symptoms.

Study Design: Prospective observational study.

Methods: One hundred ninety-two patients with clinically definite MD participated in this study. The degree of ELH was visualized by locally enhanced inner ear magnetic resonance imaging. The occurrence and intensity of vertigo attacks, hearing loss, tinnitus, and aural fullness were documented in patient diaries.

Results: There was no significant correlation between the extent of cochlear or vestibular hydrops and the number of definite vertigo days, neither with regard to a short-term nor with regard to a middle-term time period. There was also no
\end{abstract}

Menière's disease (MD) is a disorder of the inner ear which is characterized by attacks of vertigo, tinnitus, and cochlear hearing loss. Clinical diagnosis of MD has been based on the AAO-HNS Guidelines 1995 (1). In 2015, a modification of these diagnostic criteria was presented by the Classification Committee of the Barany Society, which redefined the two MD categories "definite" and "probable" $(2,3)$. The histopathologic hallmark of MD is an excess of fluid in the endolymph space of the inner ear (4), even though the role of endolymphatic hydrops (ELH) in the pathophysiology of the disease remains unclear. Recently, magnetic resonance imaging (MRI) has been used to visualize endolymphatic hydrops in MD patients in vivo, using the intratympanic $(5,6)$ or the intravenous $(7)$

\footnotetext{
Address correspondence and reprint requests to Claudia Jerin, M.D., German Center for Vertigo and Balance Disorders and Department of Otorhinolaryngology-Head and Neck Surgery, University of Munich, Grosshadern Medical Center, Marchioninistr. 15, 81377 Munich, Germany; E-mail: claudia.jerin@med.uni-muenchen.de C.J. and S.F. contributed equally.

This study was supported by the Federal German Ministry of Education and Research (grant No. 01EO1401).

The authors disclose no conflicts of interest.

DOI: $10.1097 / M A O .0000000000001681$
}

correlation between the extent of ELH and the intensity or activity of the coexisting aural symptoms hearing loss, tinnitus, and aural fullness. The duration of the disease significantly correlated with the extent of both cochlear and vestibular hydrops, but not with the number of definite vertigo days.

Conclusion: The ELH was progressive in the long-term course of the disease in this large population of definite MD patients, but short-term and middle-term fluctuations of the symptom severity did not involve measurable variations of the ELH. Furthermore, the symptom severity did not decrease with increasing disease duration. Key Words: Endolymphatic hydrops-LEIM-Menière's disease-Menière's symptoms.

Otol Neurotol 39:e123-e130, 2018. route of contrast agent application. This method enables diagnostic certainty which hitherto could only be achieved by histological postmortem examination (1), and has been used to improve differential diagnosis between MD and vestibular migraine $(8,9)$, to revise the clinical classification of hydropic ear disease (10), and to introduce new diagnostic functional test methods for MD (11). Furthermore, inner ear MRI allows for quantitative assessment of endolymphatic hydrops.

Previous studies investigating the impact of the extent of endolymphatic hydrops on audiovestibular function tests have shown a correlation between the degree of endolymphatic hydrops and the degree of hearing loss and a trend toward a correlation between the degree of endolymphatic hydrops and canal paresis measured by caloric irrigation $(6,12,13)$. It is, however, not yet clear in which way the degree of endolymphatic hydrops affects the severity of patients' symptoms. In this prospective observational study, we therefore recorded the occurrence and intensity of vertigo attacks and coexisting aural symptoms by the use of patient diaries in a large population of Menière's patients and analyzed the degree of cochlear and vestibular endolymphatic hydrops by the use of inner ear MRI. 
TABLE 1. Characteristics of the study population $(n=192)$

\begin{tabular}{lc}
\hline Male/Female & $104 / 88$ \\
\hline Age (y) & $54.3 \pm 15.3$ (range 15-79) \\
Stage: 1 & $\mathrm{n}=41$ \\
$\quad \mathrm{n}=40$ \\
3 & $\mathrm{n}=74$ \\
$\quad \mathrm{n}=37$ \\
Disease duration (mo) & $123 \pm 122$ (range 4-700) \\
Pure tone average 0.5-3 kHz (dB) & $48.4 \pm 26.3$ (range 6-130) \\
Caloric test canal paresis $(\%)$ & $22.3 \pm 31.2$ (range 0-100) \\
Vertigo days per month & $3.7 \pm 5.8$ (range 0-31) \\
Vertigo days per week & $1.0 \pm 1.7$ (range 0-7) \\
\hline
\end{tabular}

Staging was based on the pure-tone average at $0.5-3 \mathrm{kHz}$ (AAOHNS 1995).

Values are expressed as mean $\pm \mathrm{SD}$.

The aim of this study was to characterize the short-, middle-, and long-term occurrence of vertigo attacks in Menière's disease and to investigate the relationship between the extent of endolymphatic hydrops and the severity of symptoms.

\section{METHODS}

\section{Patients}

The study was conducted at a large tertiary referral center for vestibular disorders. One hundred ninety-two consecutive patients with clinically definite MD (according to the AAOHNS 1995 criteria) who underwent locally enhanced inner ear MRI (LEIM) in the 5-year period from 2011 to 2015 participated in this study (104 male $/ 88$ female, mean age $=54.3 \mathrm{y}$, age range $=15-79 \mathrm{y}$ ). These patients represented an average population of Menière's patients including different MD stages. Clinical characteristics of the study population are summarized in Table 1. Endolymphatic hydrops were visualized by LEIM after intratympanic application of contrast agent as previously described (6). Grading of the severity of cochlear and vestibular ELH on a scale of 0 to 3 was performed as described previously (6): 0: no hydrops, 1: mild hydrops, 2: marked hydrops, 3: extreme hydrops. Exemplary MR images are shown in Figure 1. One day before LEIM, all the patients underwent audiometry and caloric irrigation. Audiometry results (pure-tone average) represent the arithmetic mean of hearing thresholds at $0.5,1,2$, and $3 \mathrm{kHz}$. Caloric irrigation was evaluated on the basis of the degree of canal paresis (\%) according to the Jongkee's formula. The study protocol was approved by the institutional review board (Protocol No. 210-12) and all subjects gave written informed consent before participation in the study.

\section{Record of Symptom Severity}

All the participants were instructed to keep a daily vertigo diary to document occurrence and intensity of MD attacks, as described previously $(14,15)$. Intensity of vertigo was measured on a five-point scale ranging from zero (no vertigo) to four (worst vertigo ever): 0: vertigo-free day, 1: day with a mild attack, 2: moderately severe attacks lasting more than 20 minutes, 3: severe attacks lasting an hour or more or accompanied by nausea or vomiting, 4 : worst attack ever experienced. A definite vertigo day was defined as any day with a vertigo severity score of two or higher. Vertigo diaries were recorded by the participants for the 3 -month period 2 months before

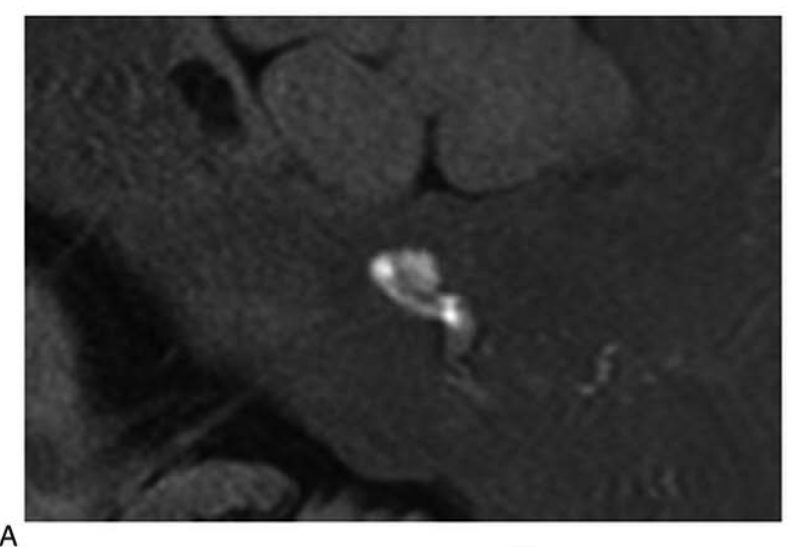

A

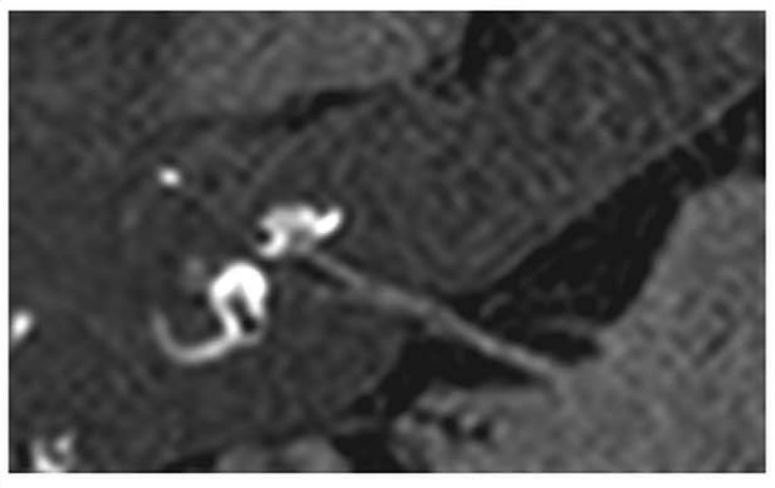

B
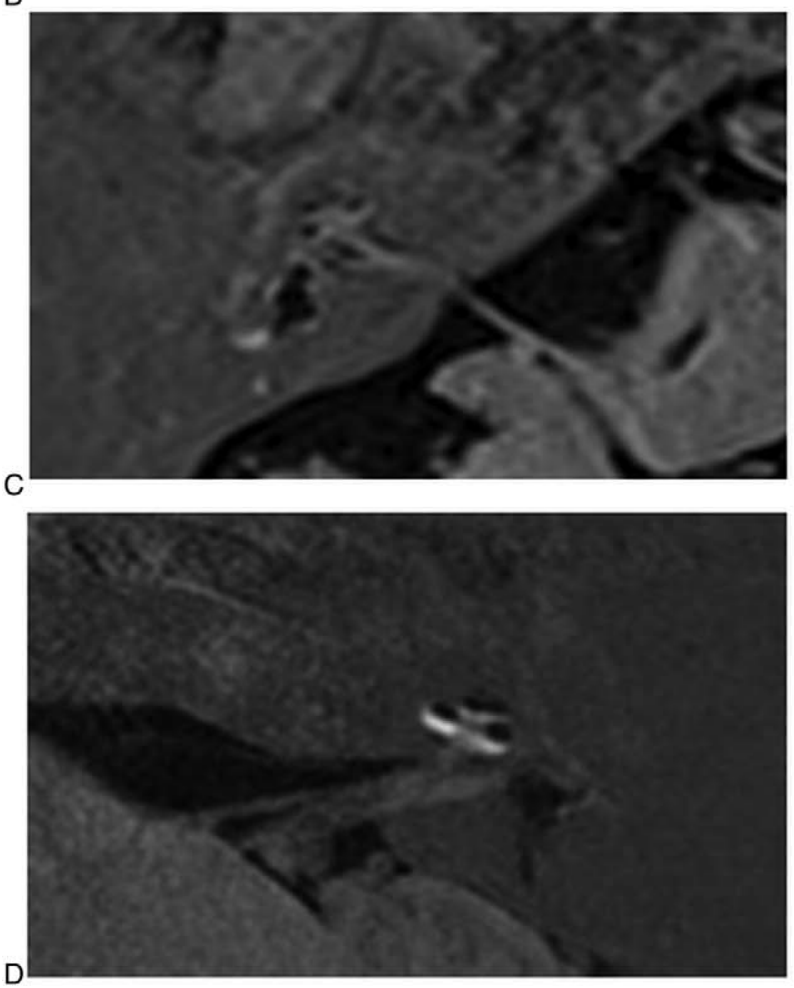

FIG. 1. Locally enhanced inner ear MR imaging with evidence of no $(A)$, mild $(B)$, marked $(C)$, and extreme (D) endolymphatic hydrops. Bright areas represent the contrastenhanced perilymphatic space, dark areas within the inner ear represent the endolymphatic space. MR indicates magnetic resonance. 

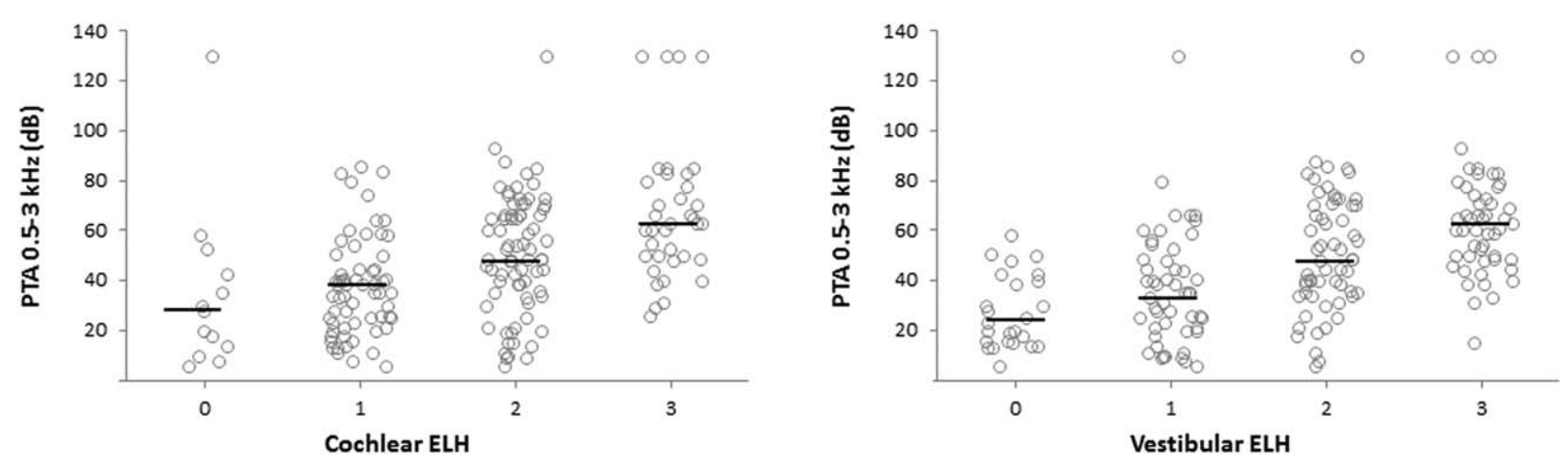

FIG. 2. Relationship between the grade of endolymphatic hydrops and pure-tone average hearing levels at $0.5,1,2$, and $3 \mathrm{kHz}$. The correlation is highly significant for both cochlear and vestibular ELH $(p<0.01)$. Horizontal bars represent the median. ELH indicates endolymphatic hydrops.

LEIM until 1 month after LEIM. Furthermore, patients were instructed to keep a more detailed and comprehensive symptom diary for the 2-week period 1 week before LEIM until 1 week after LEIM. In addition to the occurrence and intensity of MD attacks, the intensity of hearing loss, tinnitus, and aural fullness were documented in these symptom records by the use of a fivepoint scale ranging from zero to four, analogous to vertigo severity. To analyze not only the absolute symptom load, but also the symptom fluctuation as a marker of disease activity, fluctuation of symptoms was quantified by adding up the increase of severity scores from day to day during the 2-week period. In this way, fluctuation scores were calculated for vertigo, hearing loss, tinnitus, and aural fullness.

\section{Data Analysis}

Data analysis was performed with IBM SPSS Statistics 23 (SPSS Inc., Chicago, IL). The data were tested for normal distribution with the Kolmogorov-Smirnov test. Correlations between the degree of ELH, the number of definite vertigo days, fluctuation scores, disease duration, and audiovestibular function tests were calculated using the Spearman's rank correlation coefficient for nonparametric variables. Statistical significance was determined at the $p<0.05$ level.

\section{RESULTS}

Cochlear hydrops was found in 175 patients. Sixtythree patients were classified as cochlear hydrops grade
1, 75 patients as grade 2, and 37 patients as grade 3. Vestibular hydrops was detected in 161 patients. Fifty patients had vestibular hydrops grade 1, 60 patients grade 2 , and 51 patients grade 3 . There was a highly significant correlation between both cochlear and vestibular ELH and PTA (rho $=0.444, p<0.001$ and rho $=0.536$, $p<0.001$, respectively) (Fig. 2), but not between cochlear and vestibular ELH and caloric irrigation canal paresis ( $p=0.12$ and $p=0.14$, respectively) (Fig. 3).

\section{Effects of Short-term Symptom Severity}

The 2-week symptom diary served as short-term measure of symptom severity and fluctuation. On average, patients experienced 1.0 definite vertigo days per week in this period. There was no significant correlation between the number of definite vertigo days per week and the extent of cochlear or vestibular hydrops $(p=0.60$ and $p=0.06$, respectively) (Fig. 4). There was also no correlation between the extent of cochlear or vestibular hydrops and the intensity of the coexisting aural symptoms hearing loss, tinnitus, and aural fullness ( $p=0.84$, $0.65,0.67$ and $p=0.85,0.58,0.48$, respectively). Furthermore, neither cochlear nor vestibular hydrops correlated with the fluctuation of vertigo, hearing loss, tinnitus, and aural fullness $(p=0.65,0.72,0.86,0.07$ and $p=0.08,0.91,0.65,0.60$, respectively) (Fig. 5).
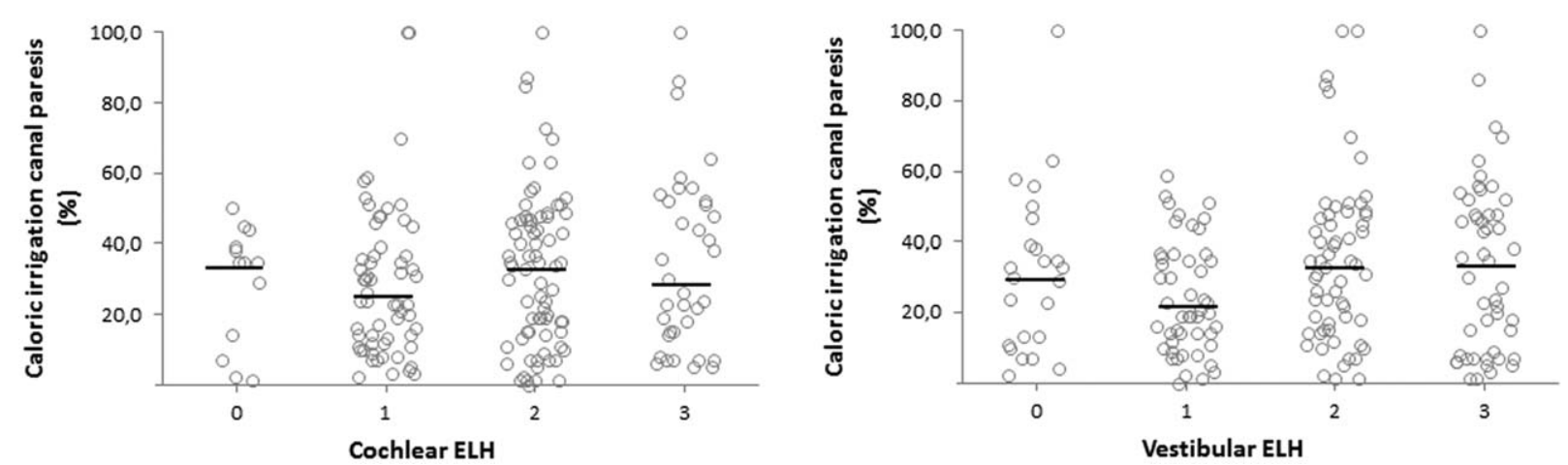

FIG. 3. Relationship between the grade of endolymphatic hydrops and horizontal semicircular canal paresis determined on caloric irrigation. There was no significant correlation. Horizontal bars represent the median. 

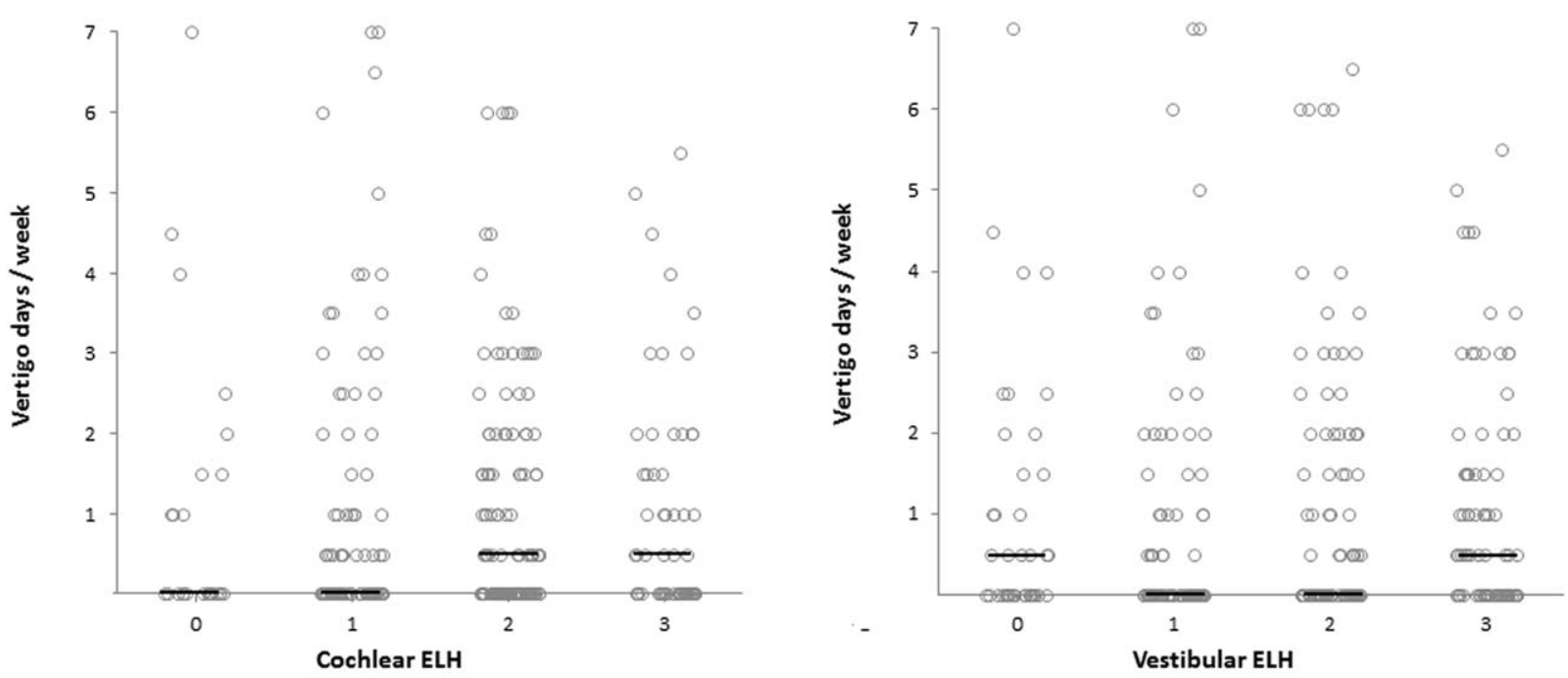

FIG. 4. Relationship between the grade of cochlear and vestibular hydrops and the number of vertigo days per week during the short-time 2-week period. There was no significant correlation. Horizontal bars represent the median.

\section{Effects of Middle-term Symptom Severity}

As regards the middle-term symptom severity, the occurrence of vertigo attacks was documented for the 3-month period 2 months before LEIM until 1 month after LEIM. The average number of definite vertigo days per month amounted to 3.7. There was no significant correlation between the number of monthly definite vertigo days and the extent of cochlear or vestibular hydrops ( $p=0.94$ and $p=0.36$, respectively) (Fig. 6). Furthermore, there was no correlation between monthly definite vertigo days and the caloric test canal paresis $(p=0.34)$. There was, however, a nonsignificant trend toward poorer audiometry results with a higher number of monthly definite vertigo days $(p=0.07)$.

\section{Long-term Effects}

The duration of the disease, i.e., the time period since the onset of audiovestibular symptoms, ranged between 4 and 700 months (average $10.3 \mathrm{y}$ ). There was a significant correlation between the duration of the disease and the extent of both cochlear and vestibular hydrops $($ rho $=0.174, p=0.03$ and rho $=0.267, p=0.001)$ (Fig. 7), as well as between disease duration on the one hand and audiometry on the other hand (rho $=0.278$, $0.278, p<0.001)$. There was no correlation between the duration of the disease and caloric irrigation $(p=0.24)$ or between the duration of the disease and the number of monthly definite vertigo days ( $p=0.43$ ) (Fig. 8).

\section{DISCUSSION}

Until a couple of years ago, the diagnosis Menière's disease was reliant on clinical criteria and the exclusion of differential diagnoses. These criteria, however, imply diagnostic uncertainty in patients who do not present with the typical symptom complex. Indeed, symptoms may distinctly vary in severity, particularly in the early stages of the disease (16). MRI-based visualization of endolymphatic hydrops after intratympanic or-because of the simpler procedure more readily available-intravenous application of contrast agent does not only allow for certain diagnosis of $\mathrm{MD}$, but also for quantitative assessment and grading of endolymphatic hydrops. Over the last 10 years, since the first report by Nakashima et al. (5) in Japan, this method has been practiced in a variety of countries worldwide, including (but not limited to!) Finland, Germany, Italy, Switzerland, the United States, Australia, China, France, Spain (17-22). Of interest, a large multicenter study confirmed that, if examined by inner ear MRI, all patients fulfilling the 1995 AAO-HNScriteria (1) of "definite MD" show evidence of endolymphatic hydrops (16).

Previously, MRI has been used to investigate whether the degree of ELH affects audiovestibular function tests. A couple of studies have shown a correlation between the extent of ELH and the degree of hearing loss and a trend toward a correlation between the extent of ELH and caloric irrigation canal paresis $(6,12,13)$. Consistent with these reports, there was a highly significant correlation between cochlear and vestibular ELH and PTA in our study cohort. However, there was no significant correlation between ELH and canal paresis, further supporting that auditory function deteriorates with increasing ELH, whereas there is no conclusive relationship between ELH and canal paresis. Other vestibular function tests like video head impulse testing, rotatory chair testing, and vestibular evoked myogenic potentials might be more strongly associated with MRI evidence of hydrops but have not been performed in our patient population.

Furthermore, we observed a highly significant correlation between the extent of cochlear and vestibular ELH and the duration of the disease, which supports the assumption that ELH progresses in the course of the disease. Evidence for a progression of ELH over time has 

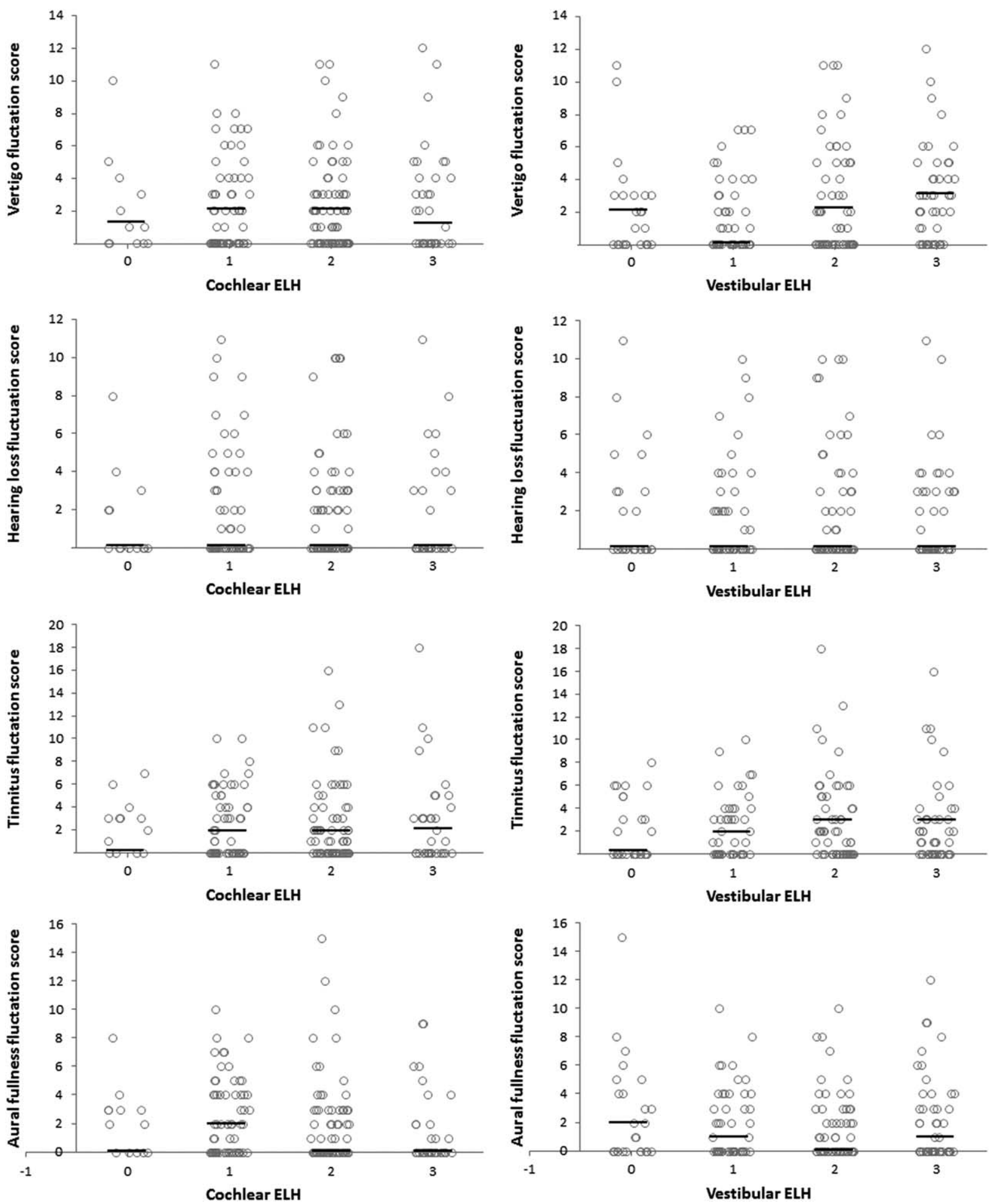

FIG. 5. Relationship between the grade of cochlear and vestibular hydrops and the fluctuation of vertigo, hearing loss, tinnitus, and aural fullness during the short-time 2-week period. There was no significant correlation. Horizontal bars represent the median.

previously been provided by studies showing a relationship between the disease duration and the degree of ELH $(6,13)$ and a study showing an enlargement of ELH in a single individual in the course of 2 years (23).
The duration of the disease, however, did not correlate with the number of monthly vertigo days, so that we did not observe any effect of disease duration on the frequency of vertigo attacks. This lack of 

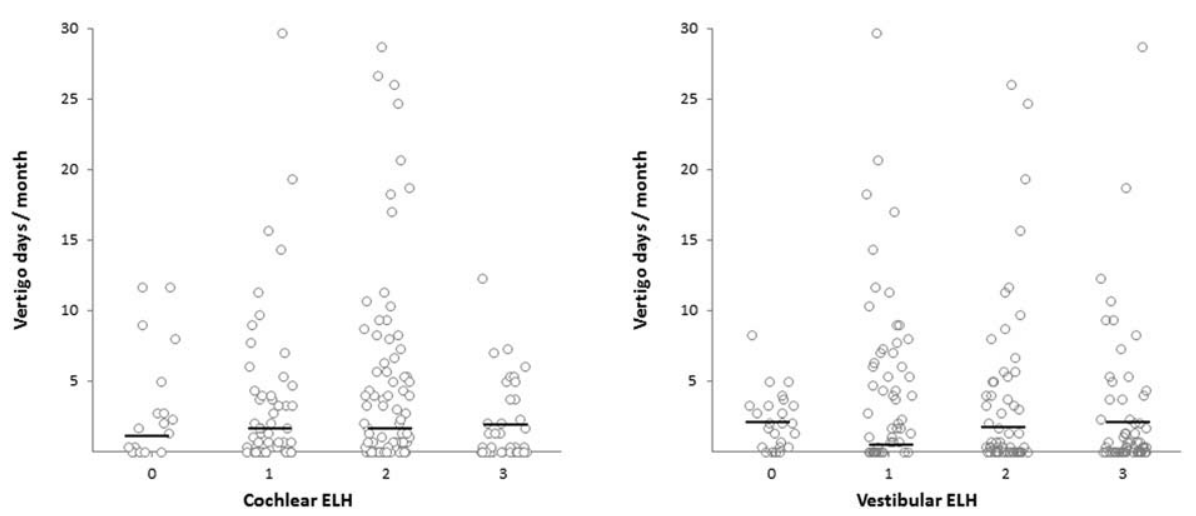

FIG. 6. Relationship between the grade of cochlear and vestibular hydrops and the number of vertigo days per month during the middletime 3-month period. There was no significant correlation. Horizontal bars represent the median.
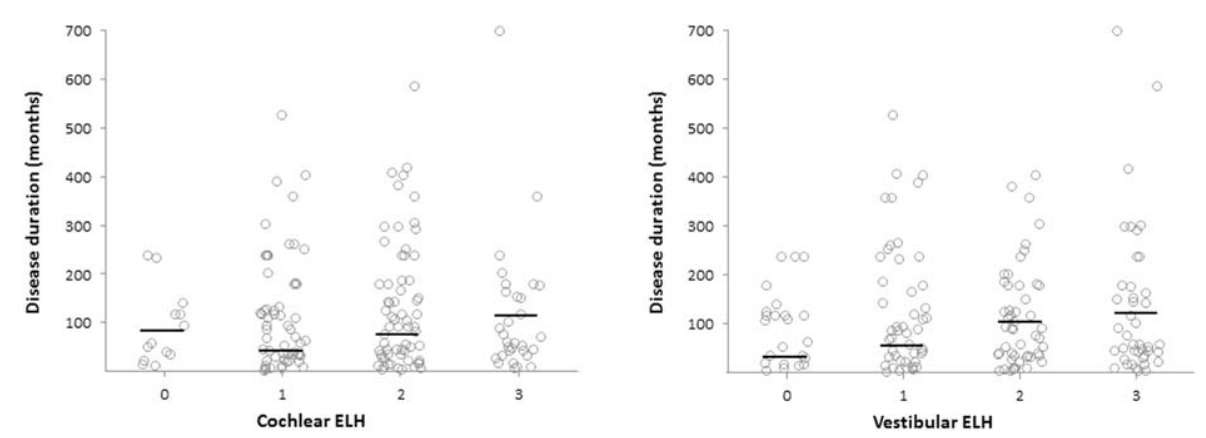

FIG. 7. Relationship between the grade of endolymphatic hydrops and the duration of the disease. The correlation is significant for both cochlear and vestibular ELH $(p=0.03$ and $p<0.01)$. Horizontal bars represent the median. ELH indicates endolymphatic hydrops.

association contradicts previous assumptions that vertiginous episodes might improve or even resolve in the course of the disease $(24,25)$, which have led to the presumptive concept of so-called "burning out" of MD. In contrast, it supports evidence that frequency and intensity of vertigo symptoms do not depend on the disease duration and that patients frequently even experience an increase of vertigo severity after longstanding disease (26).

To date, it is not known how the degree of endolymphatic hydrops affects patients' symptoms. Previous studies using the inner ear MRI to visualize ELH in smaller populations of Menière's patients found no relationship between ELH and the frequency of vertigo attacks in the months before the MRI or between hydrops and other aural symptoms such as hearing loss, tinnitus, and aural fullness $(13,27)$. To the best of our knowledge, however, this is the first study providing data from detailed symptom diaries including the occurrence and fluctuation of vertigo and concomitant aural symptoms during a short- and middle-term period in a large population of definite Menière's disease patients.
We did not observe any relationship between the extent of ELH and the frequency of vertigo attacks neither with regard to the short-term 2-week period nor with regard to the middle-term 3-month period. The severity and activity of concomitant aural symptoms were not reflected in the ELH grade, either. Short-term and middle-term fluctuations of the symptom severity

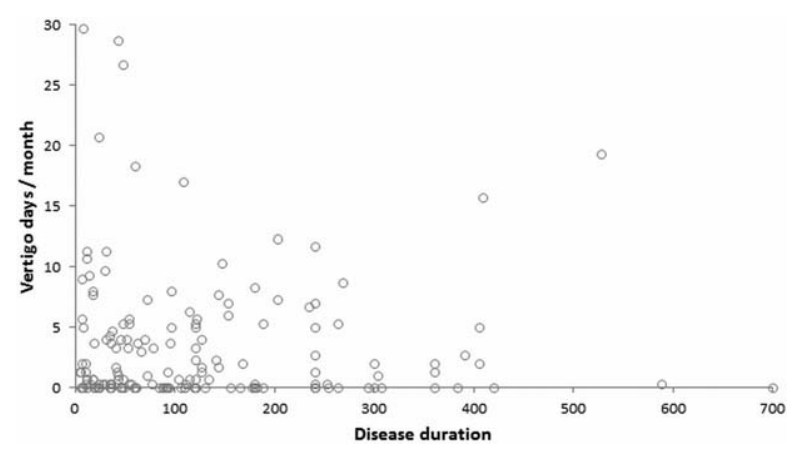

FIG. 8. Relationship between the duration of the disease and the number of monthly vertigo days. There was no significant correlation. 
thus do not seem to involve measurable variations of the ELH, even though the ELH progresses in the longterm course of Menière's disease. The lack of ELH fluctuations measurable by MRI, however, does not imply that the extent of ELH does not influence Menière's symptoms. The resolution provided by the MRI might be too low to provide visualization of slight ELH fluctuations. In other words, the slight fluctuations in ELH severity which possibly occur within a time frame of days to months may very well be of a magnitude below the resolution capacity of MR imaging. It has to be kept in mind that the normal total endolymph volume amounts to as little as $50 \mu$ l. Likewise, the endolymphatic duct of the semicircular canals is - due to its slim dimensions - not visible on MRI, in the healthy state as well as in the diseased hydropic state.

Short-term and middle-term fluctuations of the symptom severity do not entail measurable variations of the ELH. This might also implicate that there are no measurable ELH variations in the context of shortor middle-term therapeutic interventions and might provide an explanation why previous studies using inner ear MRI failed to show a reduction of the ELH following treatment of Menière's patients with betahistine, frusemide, or gentamicin $(28-30)$. These observations should be taken into consideration by future studies investigating the effect of therapeutic interventions on the ELH measured by inner ear MRI.

\section{CONCLUSION}

In this large population of definite MD patients, the endolymphatic hydrops progresses in the long-term course of Menière's disease. Hearing loss deteriorates with increasing ELH. Short-term and middle-term fluctuations of the symptom severity, in contrast, do not seem to involve measurable variations of the ELH. Furthermore, the symptom severity does not decrease with increasing disease duration.

Acknowledgments: The authors are especially grateful to Dr. Wilhelm Flatz and Prof. Birgit Ertl-Wagner for excellent collaboration in MR imaging and to Beatrice Lentge and Andrea Rosskopf for excellent technical assistance.

\section{REFERENCES}

1. AAO-HNS guidelines, 1995. Committee on Hearing and Equilibrium guidelines for the diagnosis and evaluation of therapy in Menière's disease. American Academy of Otolaryngology-Head and Neck Foundation, Inc. Otolaryngol Head Neck Surg 1995; 113:181-185

2. Lopez-Escamez JA, Carey J, Chung WH, et al. Diagnostic criteria for Meniere's disease. J Vestib Res 2015;25:1-7.

3. Goebel JA. 2015 Equilibrium Committee Amendment to the 1995 AAO-HNS Guidelines for the definition of Ménière's disease. Otolaryngol Head Neck Surg 2016;154:403-4.

4. Hallpike CS, Cairns H. Observations on the pathology of Meniere's syndrome: (Section of Otology). Proc R Soc Med 1938;31:1317-36.
5. Nakashima T, Naganawa S, Sugiura M, et al. Visualization of endolymphatic hydrops in patients with Meniere's disease. Laryngoscope 2007;117:415-20.

6. Gürkov R, Flatz W, Louza J, et al. In vivo visualized endolymphatic hydrops and inner ear functions in patients with electrocochleographically confirmed Ménière's disease. Otol Neurotol 2012; $33: 1040-5$.

7. Naganawa S, Yamazaki M, Kawai $\mathrm{H}$, et al. Visualization of endolymphatic hydrops in Ménière's disease with single-dose intravenous gadolinium-based contrast media using heavily $\mathrm{T}(2)-$ weighted 3D-FLAIR. Magn Reson Med Sci 2010;9:237-42.

8. Nakada T, Yoshida T, Suga K, et al. Endolymphatic space size in patients with vestibular migraine and Meniere's disease. J Neurol 2014;261:2079-84.

9. Gürkov R, Kantner C, Strupp M, et al. Endolymphatic hydrops in patients with vestibular migraine and auditory symptoms. Eur Arch Otorhinolaryngol 2014;271:2661-7.

10. Gürkov R, Pyykö I, Zou J, Kentala E. What is Menière's disease? A contemporary re-evaluation of endolymphatic hydrops. $J$ Neurol 2016;263 (suppl 1):S71-81.

11. Jerin C, Berman A, Krause E, et al. Ocular vestibular evoked myogenic potential frequency tuning in certain Menière's disease. Hear Res 2014;310:54-9.

12. Gürkov R, Flatz W, Louza J, et al. In vivo visualization of endolyphatic hydrops in patients with Meniere's disease: Correlation with audiovestibular function. Eur Arch Otorhinolaryngol 2011;268:1743-8.

13. Wu Q, Dai C, Zhao M, Sha Y. The correlation between symptoms of definite Meniere's disease and endolymphatic hydrops visualized by magnetic resonance imaging. Laryngoscope 2016;126: 974-9.

14. Gates GA, Green JD, Tucci DL, Telian SA. The effects of transtympanic micropressure treatment in people with unilateral Meniere's disease. Arch Otolaryngol Head Neck Surg 2004;130: $718-25$.

15. Gürkov R, Filipe Mingas LB, Rader T, et al. Effect of transtympanic low-pressure therapy in patients with unilateral Menière's disease unresponsive to betahistine: A randomised, placebo-controlled, double-blinded, clinical trial. J Laryngol Otol 2012;126:356-62.

16. Pyykkö I, Nakashima T, Yoshida T, et al. Meniere's disease: A reappraisal supported by a variable latency of symptoms and the MRI visualisation of endolymphatic hydrops. BMJ Open 2013; 3:e01555.

17. Fiorino F, Pizzini FB, Beltramello A, et al. Progression of endolymphatic hydrops in Meniere's disease as evaluated by magnetic resonance imaging. Otol Neurotol 2011;32:1152-7.

18. Barath K, Schuknecht B, Naldi AM, et al. Detection and grading of endolymphatic hydrops in Meniere disease using MR imaging. AJNR Am J Neuroradiol 2014;35:1387-92.

19. Sepahdari AR, Ishiyama G, Vorasubin N, et al. Delayed intravenous contrast-enhanced 3D FLAIR MRI in Meniere's disease: Correlation of quantitative measures of endolymphatic hydrops with hearing. Clin Imaging 2015;39:26-31.

20. Liu F, Huang W, Meng X, et al. Comparison of noninvasive evaluation of endolymphatic hydrops in Meniere's disease and endolymphatic space in healthy volunteers using magnetic resonance imaging. Acta Otolaryngol 2012;132:234-40.

21. Attye A, Dumas G, Troprès I, et al. Recurrent peripheral vestibulopathy: Is MRI useful for the diagnosis of endolymphatic hydrops in clinical practice? Eur Radiol 2015;25:3043-9.

22. Grieve SM, Obholzer R, Malitz N, et al. Imaging of endolymphatic hydrops in Meniere's disease at $1.5 \mathrm{~T}$ using phase-sensitive inversion recovery: (1) demonstration of feasibility and (2) overcoming the limitations of variable gadolinium absorption. Eur J Radiol 2012;81:331-8.

23. Jerin C, Krause E, Ertl-Wagner B, Gürkov R. Longitudinal assessment of endolymphatic hydrops with contrast-enhanced MR imaging of the labyrinth. Otol Neurotol 2014;35:880-3.

24. Green JD Jr, Blum DJ, Harner SG. Longitudinal followup of patients with Menière's disease. Otolaryngol Head Neck Surg 1991;104:783-8. 
25. Stahle J, Friberg U, Svedberg A. Long-term progression of Meniére's disease. Acta Otolaryngol Suppl 1991;485: $78-83$.

26. Havia M, Kentala E. Progression of symptoms of dizziness in Ménière's disease. Arch Otolaryngol Head Neck Surg 2004;130: 431-5.

27. Fiorino F, Pizzini FB, Beltramello A, Barbieri F. MRI performed after intratympanic gadolinium administration in patients with Ménière's disease: correlation with symptoms and signs. Eur Arch Otorhinolaryngol 2011;268:181-7.
28. Fiorino F, Pizzini FB, Barbieri F, Beltramello A. Magnetic resonance imaging fails to show evidence of reduced endolymphatic hydrops in gentamicin treatment of Ménière's disease. Otol Neurotol 2012;33:629-33.

29. Gürkov R, Flatz W, Keeser D, et al. Effect of standard-dose Betahistine on endolymphatic hydrops: An MRI pilot study. Eur Arch Otorhinolaryngol 2013;270:1231-5.

30. Fiorino F, Mattellini B, Vento M, et al. Does the intravenous administration of frusemide reduce endolymphatic hydrops? $J$ Laryngol Otol 2016;130:242-7. 